\title{
A Novel Biphasic Scaffold with Gradient Pore Structures for Osteochondral Tissue Engineering
}

\author{
Duo PAN \\ Complex and intelligent research center \\ East China University of Science and Technology \\ Shanghai, P.R. China, \\ e-mail: Dora_PD@163.com \\ Hongbo ZHANG* \\ Complex and intelligent research center \\ East China University of Science and Technology \\ Shanghai, P.R. China, \\ e-mail: hbzhang@ecust.edu.cn
}

\author{
Ruixue YIN \\ Complex and intelligent research center \\ East China University of Science and Technology \\ Shanghai, P.R. China, \\ e-mail: yinruixue@ecust.edu.cn \\ Shengbing YANG \\ Shanghai Key Laboratory of O Orthopaedic Impact \\ Shanghai Ninth People's Hospital \\ Shanghai, P.R. China \\ e-mail: bioshengbingy@163.com
}

\author{
Wenjun ZHANG \\ Department of Mechanical Engineering \\ University of Saskatchewan \\ Canada \\ e-mail: wjz485@mail.usask.ca
}

\begin{abstract}
This study was aimed at using biomimetic strategies to construct a biphasic scaffold. Biphasic scaffolds were constructed using the 3-dimentional printing technology with two phases: the bony phase and the chondral phase. The bony phase was using the composition of poly (lactide-co-glycolide) (PLGA) and Polyethylene glycol PEG; the chondral phase was using hydrogel consisting of GelMA and hyaluronic acid. Also we designed a gradient pore size in the bony phase to better mimic the natural bone tissue. In vitro tests have shown the stratified scaffolds with gradient designs were effective for the multi-tissue formation.
\end{abstract}

Keywords-gradient scaffold; 3D printing

\section{INTRODUCTION}

Articular cartilage defects in joints can hardly be cured due to its avascular character and lack of osteocytes and chondrocytes. Recently researchers point out that osteochondral (OC) tissue engineering would be an effective way for the treatment of this disease by reconstructing cartilage scaffold including cartilage, subchondral bone and the contact interface between bone and cartilage. This kind of scaffold can offer the similar structure, function, tissue performance as the nature OC tissue [1].

The nature OC tissue has a hierarchical organization. There are three functional grading layers: the soft cartilage, the stiff underlying bone and an interface between the two layers [2]. To maintain the appropriate connections, nature tissues usually show a progressive change in both structure and composition. Porous materials and gradient scaffolds are important to mimic nature tissue. Hydrogels have been widely used for osteochondral regeneration due to the ability to provide biochemical signals (e.g., cellular differentiation and migration) [3, 4]. Derived from collagen, gelatin is soluble in water and presents several advantages for cartilage repair: gelatin is a biocompatible, biodegradable material, and it has no antigenicity in physiological conditions. Compared to collagen, gelatin is with low cost [5]. Hyaluronic acid (hyaluronic acid, HA) is a natural polymer linear polysaccharide, exists in extracellular matrix of cartilage [6]. When mixed with gelatin, hyaluronic acid improves the biomechanical ability to maintain spatial and temporal control during healing. However, hydrogels alone in osteochondral regeneration still have some limitations due to the fast degradation and poor mechanical strength of hydrogels. Poly (lactide-co-glycolide) (PLGA) is the most widely used biodegradable materials in preclinical studies of ligament, tendon and cartilage, as well as in bone regenerative medicine [7]. This is because PLGA scaffolds are porous and suitable for the attachment, proliferation and differentiation of cells without affecting their morphology and growth. Moreover, the scaffolds possess better mechanical property and are readily fabricated [8]. Polyethylene glycol (PEG) has been proved to achieve a range of mechanical properties which are suitable for cell encapsulation $[9,10]$.

In the past few years, a number of scaffolds have been fabricated. Researchers tend to investigate the influence of pore size on cell proliferation and differentiation. Cells respond differently to different pore size and the porosity 
affects the transport of nutrients, wastes and cellular signals during the tissue regeneration. For most of the porous scaffolds, pore size and porosity have great effect on cell attachment, growth, proliferation and differentiate [11]. Zhao et al. [12], Zhang et al.[13], Oh et al.[14], Huri et al.[15] prove cell behavior can be affected by the pore size, but there are still no convincing conclusions illustrating the relationship between pore size and cell behavior. Swieszkowski et al. [16] developed two kinds of hybrid scaffolds via fused deposition modeling then seeded bone marrow mesenchymal cells (MSC) into the scaffolds. Their study showed that tissue engineering has big impact in the field of cartilage repair in the future. A novel composite, the biphasic scaffold technology with a MSC, has been shown a high potential in the knee defect regeneration. However, several problems still need to be solved for the clinical application, such as scaffold design, cellular delivery and implantation strategies.

In previous researches, lots of methods have been used to fabricate porous scaffold such as porogen leaching, freeze-drying and centrifugation method, but all the above-mentioned production processes cannot accurately control the distribution and diameter size of pores in the scaffolds.

Three-dimensional printing, a rapid prototyping method, provides many advantages such as precise localization ability and convenient controlling performance. The 3D printing technology is considered as a promising way to investigate how the pore sizes effect on cell behavior. In this paper, hybrid scaffolds with a gradient structure were fabricated using the 3D printing technology. We formed a continuous system using PLGA/PEG as stiff bony phase with different pore grade and gelatin/hyaluronic acid as soft cartilage-like phase, subsequently seeded mouse mesenchymal stem cells and chondrocytes respectively. Such osteochondral scaffolds with the gradient material and structure provided a stable biotic environment for cell adhesion, proliferation and differentiation.

\section{MAterials AND MEthods}

PLGA with a lactide/glycolide ratio of 75/25 (Mw: 70 kDa; Sigma) and 5\% content PEG (Mw: 44.05, Mn:6000, Aladdin)were dissolved in dichloromethane (AR,99.5\%, Aladdin).The solvent was evaporated in a vacuum drying oven for $12 \mathrm{~h}$ at $45^{\circ} \mathrm{C}$. Hydrogels containing 5\%wt/V GelMA, 3\%wt/V hyaluronic acid (Mw: 100kDa, Aladdin) and $1 \%$ owt $/ \mathrm{V}$ photoinitiator (I-2959, (2-hydroxy-1-[4-(2-hy-droxyethoxy)phenyl]-2-methyl-1-pro panone) (Ciba Speciality Chemicals Corp., Tarrytown, NY) were dissolved at $37^{\circ} \mathrm{C}$.

Mouse mesenchymal stem cells (mMSCs) and chondrocytes were kindly provided by Shanghai ninth people's hospital and cultured in basal medium: Dulbecco minimum essential medium (DMEM) supplemented with $10 \%$ fetal calf serum (FCS), and 1\% antibiotics and 1640 supplemented with $10 \%$ fetal bovine serum (FBS), and $1 \%$ antibiotics respectively, and incubated at $37^{\circ} \mathrm{C}$ in a humidified 95\%air/5\%CO2 atmosphere (standard condition).

\section{A. Multi-layered Scaffold Fabrication}

3D bio-plotter (Envision TEC, Germany) was successfully directly applied to produce novel 3D porous hybrid scaffolds with fully interconnected porous networks and highly controllable porosity and pore size. The bio-plotter possessed one low and one high temperature head. Hybrid scaffolds were printed layer by layer to the size of $8 \mathrm{~mm}$ in diameter, $1.5 \mathrm{~mm}$ in depth and arrayed in a quadrilateral pattern. The bottom six layers of the scaffold were designed to be printed with PLGA/PEG using high temperature head as Fig. 1a, and the top two layers were printed with hydrogel using low temperature head. In the printing process, PLGA/PEG was heated to $180^{\circ} \mathrm{C}$, and then printed of different fiber spacing from $600 \mu \mathrm{m}$ to $400 \mu \mathrm{m}$ as bony phase (1.2 mm in depth). Hydrogel was heated to $37^{\circ} \mathrm{C}$, uniformly printed on the bone phase $(0.3 \mathrm{~mm}$ in depth). PLGA/PEG scaffolds with six layers uniform pore sizes were printed as control group shown in the Fig. 1b. All printing parameters such as the pressure and velocity of the nozzle were adjusted according to material properties and working conditions.

(a)

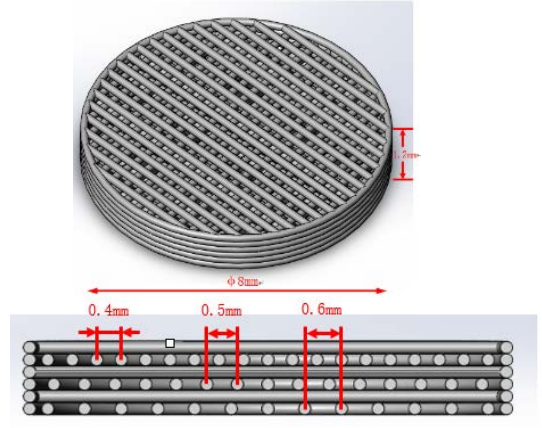

(b)

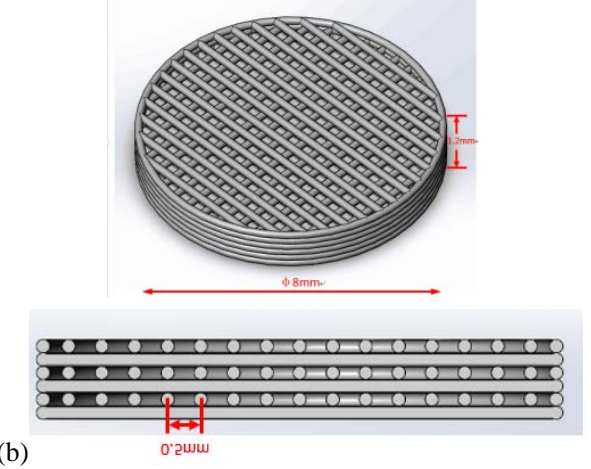

Figure 1. The schematic diagram of printing model bony phase of biphasic scaffold. (b) uniform scaffold

\section{B. Scanning Electron Microscopy (Sem)}

Scanning electron microscopic (SEM; JSM-6360LV) analysis was performed to detect the microstructure of hybrid scaffolds.

\section{Mechanical Tests}

Mechanical tests were conducted on a universal testing machine (GOTECH TESTING MACHINES DONG GUAN CO., LTD) to access the compressive properties of hybrid 
scaffolds. Compressive tests ( $\mathrm{n}=5$ /group) were performed at a constant strain rate of $1 \mathrm{~mm} / \mathrm{min}$ to $70 \%$ strain $(1.5 \mathrm{mN}$ pre-load) under room temperature. The compressive modulus was calculated from the slope of stress-strain curve.

\section{Live/Dead Staining}

Cell adhesion and spreading was evaluated using a live/dead cell assay kit (Thermo Fisher Scientific, USA) at 3 days after cell seeding. $2 \mu \mathrm{l}$ EthD-1 stock solution (component B) and $0.5 \mu \mathrm{l}$ calcein AM stock solution (component A) were mixed with $1 \mathrm{ml}$ phosphate buffer saline (PBS). Added $200 \mu \mathrm{l}$ combined Live/dead assay to each well making sure the cells were covered with the solution. Then cells were incubated for $30 \mathrm{~min}$ in incubator $\left(37^{\circ} \mathrm{C}, 5 \% \mathrm{CO}_{2}\right)$ and observed under confocal microscope (VK-X100K, Kevence, Japan).

\section{E. CCK-8 Assay}

Cell counting kit-8 was applied to detect cell proliferation at days 1,4 , and 7 . For cell proliferation and cytotoxicity assay, each scaffold sample was seeded mMSCs or chondrocytes $5 \times 10^{4}$ cells per well in 48 well-plates and incubated in incubator $\left(37^{\circ} \mathrm{C}, 5 \% \mathrm{CO}_{2}\right)$ for up to 7 days, cell culture medium was changed every 3 days. CCK-8 solution was diluted with DMEM to $10 \%$ each well. After 2 hours incubation, the absorbance was measured at $450 \mathrm{~nm}$ using a microplate reader (TECAN GENIOS Pro).

\section{F. Osteogenic Differentiation of mMSCs}

$3 \times 10^{4}$ mMSCs per well were seeded on each sample to test the effect of scaffolds with gradient pore size on the osteogenic differentiation. After 48 hours incubation, the culture medium in 48 well-plates was changed into osteogenic induction medium. The medium was prepared basing on human mesenchymal stem cell osteogenic differentiation basal medium, with $20 \mathrm{~mL}$ FBS, $2 \mathrm{~mL}$ penicillin-streptomycin, $2 \mathrm{~mL}$ glutamine, $400 \mu \mathrm{L}$ ascorbate, $2 \mathrm{~mL} \beta$-glycerophosphate, and $20 \mu \mathrm{L}$ dexamethasone. The osteogenic induction medium was changed every two days. After 7, 10, 14 days incubation, the alkaline phosphatase (ALP) activity and staining was accomplished following the procedure in the protocol of Alkaline phosphatase assay kit(Jiancheng Bioengineering Institute, Nanjing, China) and BCIP/NBT ALP staining kit (Biyuntian, Shanghai, China) respectively.

\section{G. Statistical Analysis}

Statistical analysis was performed using the SPSS 13.0. All the numerical results were presented as mean \pm standard deviation. T-test was used respectively. A level of $\mathrm{P}<0.05$ was considered statistically significant

\section{RESULTS}

\section{A. Scaffolds Characterization}

PLGA/PEG scaffolds of $8 \mathrm{~mm}$ in diameter, $1.2 \mathrm{~mm}$ in depth were printed as shown in Fig. 2. Fig. 2a showed the uniform pore size. Each fiber aligned orderly and the fiber spacing among each layer was consistent. Fig. $2 \mathrm{~b}$ showed the gradient design, an obvious fiber interlacing could be seen from the surface and from the cross section, fiber spacing graded arranged. The porosity of the gradient and uniform scaffold was $\sim 62.8 \%$ and $\sim 60 \%$ respectively.

(a)

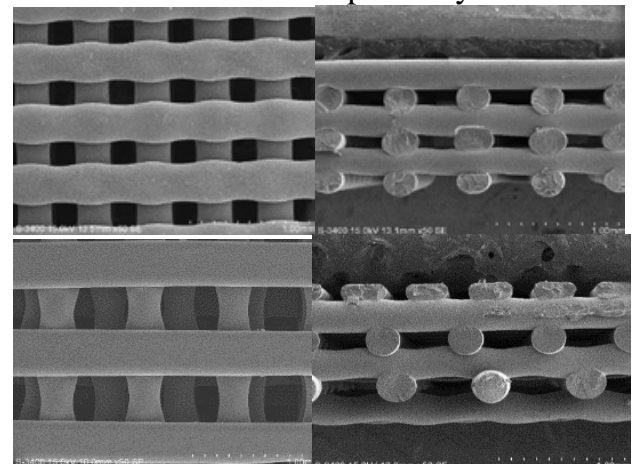

(b)

Figure 2. Surface and cross section SEM of PLGA/PEG phase (a) uniform structure with pore size around $0.3 \mathrm{~mm}$ (b) gradient structure with pore size $0.6 \mathrm{~mm}, 0.5 \mathrm{~mm}, 0.4 \mathrm{~mm}$ from bottom to top

The stress-strain curve of compressive tests was shown in Fig. 3. Compressive modulus Es was calculated from the tangent curve, for gradient structure Es $=18 \mathrm{MPa}$, and for uniform control group Es $=21 \mathrm{MPa}$. The compressive modulus for native meniscus was around 56MPa, and for cartilage was around 12MPa. Although the compressive modulus of scaffolds with uniform structure was higher than those with gradient structure, they both matched nature biomechanics of human environment.

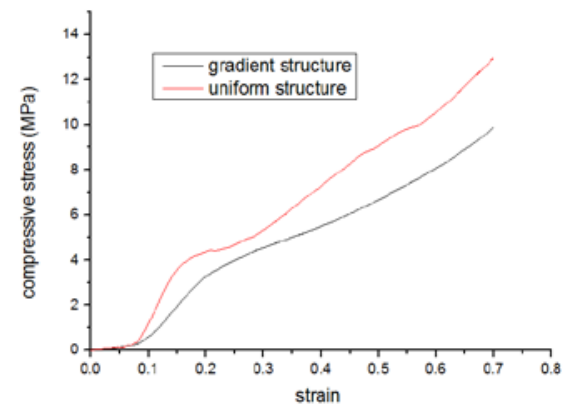

Figure 3. Average stress-strain curve $(n=5)$

\section{B. Adhesion and Proliferation of Cells on Multi-layered Scaffolds}

Cell adhesion and spreading behavior was important parameter to test the interaction between cell and scaffold. Fig. 4 showed the mMSCs on scaffolds stained with DAPI (4', 6-diamidino-2-phenylindole) and FITC-phalloidin after 24 hours culture. Confocal laser staining microscope photos showed that after 24 hours, the cell nucleus were like spheroidicity, and cell actin were fully spreading, there were no cell boundary on the edge. Cells were tightly adhering on the materials. 


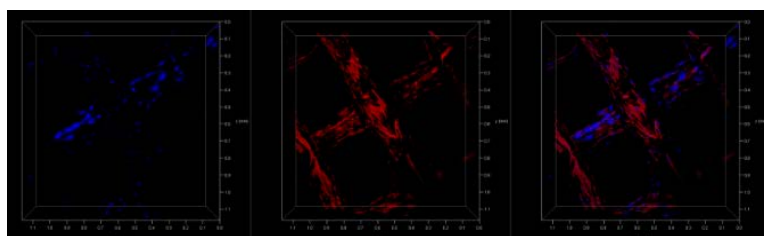

Figure 4. The confocal laser scanning microscope photos of the mMSCs cultured $24 \mathrm{~h}$ on the scaffolds.

To further test the cell viability on the scaffolds, we used live/dead staining assay. Fig. 5 showed live/dead staining of mMSCs and chondrocytes on the scaffolds cultured for 3 days. Live cells were stained with calcein AM (green color) and dead cells were stained with EthD-1 (red color). In Fig. $5 \mathrm{a}$, the green points were far more than the red ones. The percentage of cell viability for mMSCs in scaffolds was over 90\%. From the staining results of chondrocytes shown in Fig. 5b, barely dead cells can be seen, and the cell morphology was spheroidicity, the typical chondrocytes morphology.

(a)

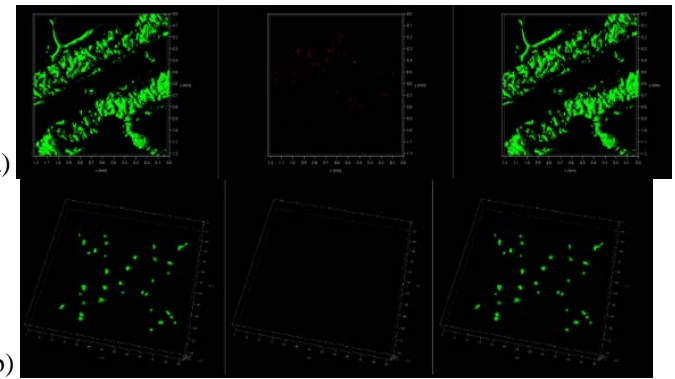

Figure 5. Live/dead staining of (a) mMSCs cultured for 3 days (b) chondrocytes cultured for 3 days

Proliferation of mMSCs and chondrocytes were evaluated with CCk-8 assay. The results were shown in the Fig.6. The proliferation assay of mMSCs and chondrocytes were performed at three time points, at 1, 4 and 7 day. The optical density (OD) value on single phase scaffolds was lower than that on hybrid scaffolds. On day 7, the growth of mMSCs was obviously greater on the hybrid scaffold than that on the pure PLGA scaffolds. For chondrocytes, the number of cells on the hybrid scaffolds was larger than that on the single phase scaffold. On day 1 and day 4, the growth of cell number was slowly, but on day 7 , there was a significant growth on both scaffolds.

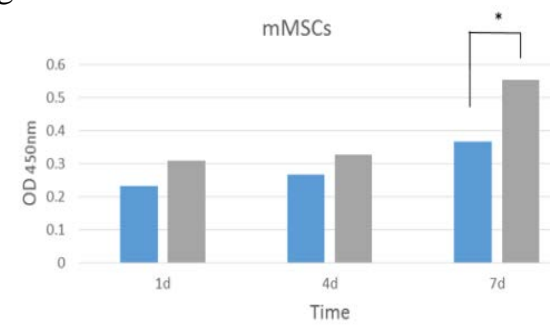

(a)

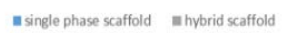

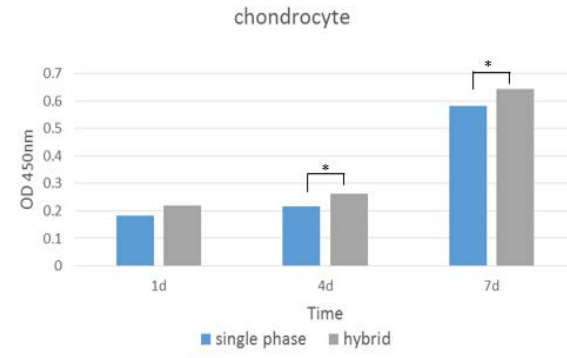

Figure 6. Proliferation of (a) mMSCs (b) chondrocytes on single phase and hybrid scaffolds $(* \mathrm{P}<0.05)$

The ALP activity in different kind of scaffolds was observed over a period of 14 days at three time points (day 7 , day 10, and day 14). Fig. 7a showed the positive ALP staining on the scaffolds at day 14, and the staining on the scaffolds with gradient pore size was significantly denser than that on the uniform structure scaffolds. In Fig. 7b, it can be observed that the mMSCs on the gradient groups showed significantly higher ALP activity than those cultured on the uniformed scaffolds at day 10 and day 14.By the 14th day, the ALP activity level of the scaffolds surfaces increased, and increase rate of ALP activity on the gradient groups was more than that on other scaffolds.

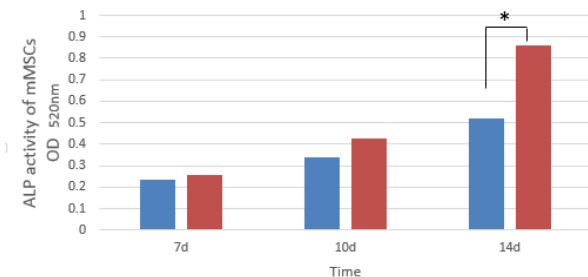

(a)

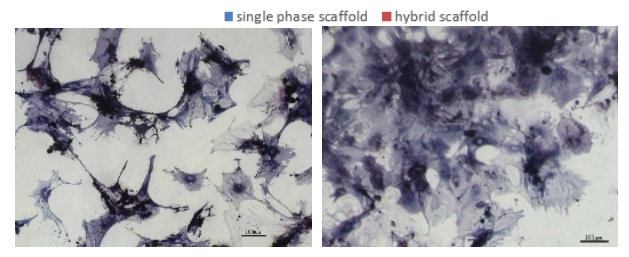

Figure 7. The ALP activity and ALP staining assay. (a) ALP activity of mMSCs after7, 10, and 14 days of culture $p<0.05$ (b) ALP staining at 14day, measuring scale: $100 \mu \mathrm{m}$

\section{CONCLUSION}

In this study, biphasic scaffolds with gradient pore size were constructed by 3D printing. Such biomimic scaffolds were proved to have enough compressive strength to support the cells surviving in the real biomechanical microenvironment and be stable for the adhesion and proliferation. Also, the biomimic scaffolds provided more a suitable environment for mMSCs differentiation than on pure PLGA scaffolds.

\section{REFERENCES}

[1] Ding C, Qiao Z, Jiang W, et al.. Regeneration of a goat femoral head using a tissue-specific, biphasic scaffold fabricated with CAD/CAM technology. Biomaterials. 2013, 34(28): 6706-6716.

[2] VL Ferguson, RC Paietta.The Bone - Cartilage Interface. 2013.

[3] Bhavita joshi, Dr.George Collins,Dr.Treena Arinzeh. Gelatin/Sulfated 
Polysaccharides Blends: An Approach to Mimic Hydrogel Behavior in Articular Cartilage. 2011

[4] Park J Y, Choi J C, Shim J H, et al. . A comparative study on collagen type I and hyaluronic acid dependent cell behavior for osteochondral tissue bioprinting. Biofabrication. 2014, 6(3): 35004.

[5] Amadori S, Torricelli P, Panzavolta S, et al.. Multi-Layered Scaffolds for Osteochondral Tissue Engineering: In Vitro Response of Co-Cultured Human Mesenchymal Stem Cells. Macromolecular Bioscience. 2015, 15(11): 1535-1545.

[6] David N. Kunz1, Daniel Aeschlimann2, Paul Bulpitt3 et al.. Biomechanical Behavior of Novel Biocompatible Hydrogels for Repair of Osteochondral Defects. Proceedings of the 22"' Annual EMBS International Conference, 2000, July 23-28, Chicago IL

[7] ImGI,AhnJH,KimSY,etal..Ahyaluronate-atelocollagen/beta-tricalcium phosphate-hydroxyapatite biphasic scaffold for the repair of osteochondral defects: a porcine study. Tissue Eng Part A. 2010,16(4): 1189-1200.

[8] Li M, Liu W, Sun J, et al.. Culturing Primary Human Osteoblasts on Electrospun Poly (lactic-co-glycolic acid) and Poly (lactic-co-glycolic acid)/Nanohydroxyapatite Scaffolds for Bone Tissue Engineering [J]. ACS Applied Materials \& Interfaces.2013, 5(13): 5921-5926.

[9] Bryant S J, Bender R J, Durand K L, et al.. Encapsulating chondrocytes in degrading PEG hydrogels with high modulus: engineering gel structural changes to facilitate cartilaginous tissue production. Biotechnol Bioeng. 2004, 86(7): 747-755.
[10] Kinneberg K R C, Nelson A, Stender M E, et al.. Reinforcement of Mono- and Bi-layer Poly (Ethylene Glycol) Hydrogels with a Fibrous Collagen Scaffold. Annals of Biomedical Engineering. 2015, 43(11): 2618-2629.

[11] Tang G, Zhang H, Zhao Y, et al.. Preparation of PLGA scaffolds with graded pores by using a gelatin-microsphere template as porogen. $\mathrm{J}$ Biomater Sci Polym Ed. 2012, 23(17): 2241-2257.

[12] Zhao Y, Tan K, Zhou Y, et al.. A combinatorial variation in surface chemistry and pore size of three-dimensional porous poly( $\varepsilon$-caprolactone) scaffolds modulates the behaviors of mesenchymal stem cells. Materials Science and Engineering: 2016, C. 59: $193-202$

[13] Zhang Q, Lu H, Kawazoe N, et al.. Pore size effect of collagen scaffolds on cartilage regeneration. Acta Biomaterialia. 2014, 10(5) 2005-2013.

[14] Oh S H, Kim T H, Im G I, et al.. Investigation of Pore Size Effect on Chondrogenic Differentiation of Adipose Stem Cells Using a Pore Size Gradient Scaffold. Biomacromolecules. 2010, 11(8): 1948-1955.

[15] Huri P Y, Ozilgen B A, Hutton D L, et al.. Scaffold pore size modulates in vitro osteogenesis of human adipose-derived stem/stromal cells. 2014, Biomed Mater. 9(4): 45003.

[16] Swieszkowski W, Tuan B.. Repair and regeneration of osteochondral defects in the articular joints. Biomolecular Engineering. 2007 